\title{
Cooperative Communications With Outage-Optimal Opportunistic Relaying
}

\author{
Aggelos Bletsas, Member, IEEE, Hyundong Shin, Member, IEEE, and Moe Z. Win, Fellow, IEEE
}

\begin{abstract}
In this paper, we present simple opportunistic relaying with decode-and-forward (DaF) and amplify-and-forward (AaF) strategies under an aggregate power constraint. In particular, we consider distributed relay-selection algorithms requiring only local channel knowledge. We show that opportunistic DaF relaying is outage-optimal, that is, it is equivalent in outage behavior to the optimal DaF strategy that employs all potential relays. We further show that opportunistic AaF relaying is outage-optimal among single-relay selection methods and significantly outperforms an AaF strategy based on equal-power multiple-relay transmissions with local channel knowledge. These findings reveal that cooperation offers diversity benefits even when cooperative relays choose not to transmit but rather choose to cooperatively listen; they act as passive relays and give priority to the transmission of a single opportunistic relay. Numerical and simulation results are presented to verify our analysis.
\end{abstract}

Index Terms-Cooperative diversity, fading relay channel, outage probability, wireless networks.

\section{INTRODUCTION}

$\mathbf{U}$ TILIZATION of terminals distributed in space can significantly improve the performance of wireless networks [1]-[3]. For example, a pair of neighboring nodes with channel state information (CSI) can cooperatively beamform towards the final destination to increase total capacity [2]. Even when CSI is not available or when radio hardware cannot support beamforming, cooperation between the source and a single relay provides improved robustness to wireless fading [3]. Basic results for cooperation are presented in [4]-[6] and references therein.

Scaling cooperation to more than one relay is still an open area of research, despite the recent interest in cooperative communication. One possible approach is the use of distributed space-time coding among participating nodes [7]. In practice, such code design is quite difficult due to the

Manuscript received February 28, 2006; revised July 31, 2006; accepted August 21, 2006. The associate editor coordinating the review of this paper and approving it for publication was A. Conti. This research was supported in part by the Kyung Hee University Research Fund in 2007 (KHU-20070773), the Office of Naval Research Young Investigator Award N00014-03-1-0489, the National Science Foundation under Grants ANI-0335256 and ECS0636519, DoCoMo USA Labs, and the Charles Stark Draper Laboratory Robust Distributed Sensor Networks Program.

A. Bletsas was with the Massachusetts Institute of Technology (MIT) Media Laboratory. He is now with the Radiocommunications Laboratory (RCL), Department of Physics, Aristotle University of Thessaloniki, Thessaloniki 54124, Greece (email: bletsas@auth.gr).

H. Shin was with the Laboratory for Information and Decision Systems (LIDS), Massachusetts Institute of Technology, Cambridge, MA 02139 USA. $\mathrm{He}$ is now with the School of Electronics and Information, Kyung Hee University, 1 Seocheon, Kihung, Yongin, Kyungki 446-701, Korea (e-mail: hshin@khu.ac.kr)

M. Win is with the Laboratory for Information and Decision Systems (LIDS), Massachusetts Institute of Technology, 77 Massachusetts Avenue, Cambridge, MA 02139 USA (e-mail: moewin@mit.edu).

Digital Object Identifier 10.1109/TWC.2007.xxxxx. distributed and ad-hoc nature of cooperative links, as opposed to codes designed for co-located multiple-input multipleoutput (MIMO) systems [8]-[10]. The need and availability of global CSI is fundamental in distributed environments. For example, additional communication is needed for each relay to acquire CSI about other relays (as needed in [11]) or for the destination to acquire CSI between the source and all relays (as needed in [12]). Moreover, the number of useful antennas (distributed relays) for cooperation is generally unknown and varying. Therefore, coordination among the cooperating nodes is needed prior to the use of a specific space-time coding scheme, typically designed for a fixed number of transmit antennas. Furthermore, it is often assumed in the literature that the superposition of signals transmitted by several relays is always constructive. ${ }^{1}$

Such assumption requires distributed phased-array techniques (beamforming) and unconventional radios, thereby increasing complexity and cost of each transmitter. Finally, coherent reception of multiple-relay (MR) transmissions requires tracking of carrier-phase differences among several transmitreceive pairs, which increases the cost of the receiver.

Therefore, simplification of radio hardware in cooperative diversity setups is important. Antenna selection, invented for classical multiple-antenna communications [14]-[18], is one approach to minimize the required cooperation overhead and to simultaneously realize the potential benefits of cooperation between multiple relays. In particular, a simple, distributed, single-relay selection algorithm was proposed for slow fading wireless relay channels [19]. This single-relay opportunistic selection provides no performance loss from the perspective of diversity-multiplexing gain tradeoff, compared to schemes that rely on distributed space-time coding.

In this paper, we present single-selection-opportunisticrelaying with decode-and-forward $(\mathrm{DaF})$ and amplify-andforward $(\mathrm{AaF})$ strategies and analyze their outage probability under an aggregate power constraint. ${ }^{2}$ The motivation behind imposing the aggregate power constraint is threefold: (i) transmission power is a network resource that affects both the lifetime of the network with battery-operated terminals and the scalability of the network; (ii) regulatory agencies may limit total transmission power due to the fact that each transmission can cause interference to the others in the network; and (iii) cooperative diversity benefits can be exploited even when relays do not transmit (and therefore, do not add transmission energy into the network). We consider both reactive and proactive relay selection depending on whether the relay selection is performed after or before the source transmission.

\footnotetext{
${ }^{1}$ This case includes Gaussian relay channels where propagation coefficients are assumed to be real numbers [13].

${ }^{2} \mathrm{The} \mathrm{DaF}$ strategy is also known as regenerative processing.
} 


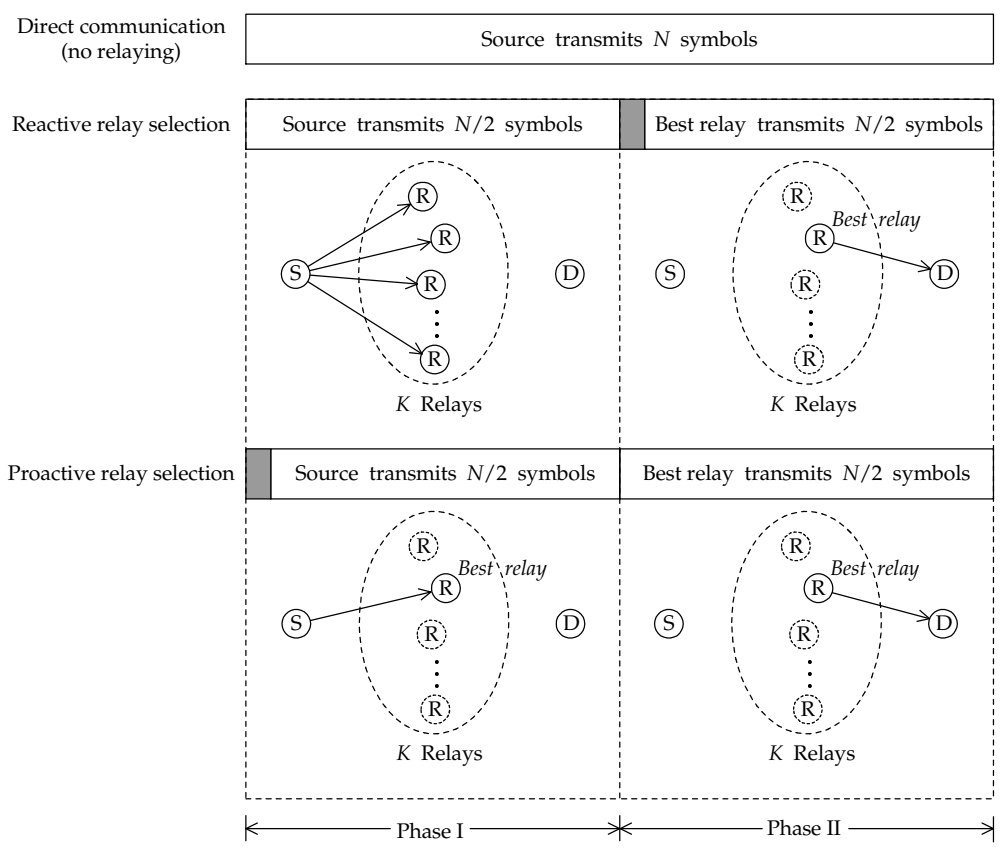

Fig. 1. A half-duplex dual-hop communication scenario: the source and destination are blocked or have poor connection. Opportunistic relay selection can be performed proactively before the source transmission or reactively after the source transmission. The shaded band indicates when relay selection occurs.

The contributions of this paper are as follows.

- We propose simple opportunistic relaying schemes with $\mathrm{DaF}$ and $\mathrm{AaF}$ strategies, which can be performed in a distributed manner without requiring global CSI at each relay or at a central controller in the network, thereby reducing the required cooperation overhead.

- We show that both reactive and proactive opportunistic $\mathrm{DaF}$ relaying are outage-optimal, that is, they are equivalent in outage behavior to the optimal DaF strategy that employs all potential relays.

- We show that opportunistic AaF relaying is outageoptimal among single-relay selection methods. Additionally, opportunistic AaF significantly outperforms an $\mathrm{AaF}$ strategy based on equal-power MR transmissions, when only local CSI is available.

- Proactive opportunistic relay selection allows all relays, except a single opportunistic relay, to enter an idle mode during the source transmission, thereby reducing the reception energy cost in the network.

These results reveal that relays are useful even when they do not actively transmit, provided that they adhere to the "opportunistic" cooperation rule and give priority to the "best" available relay. The simplicity of our protocol allows immediate implementation in a custom radio hardware. ${ }^{3}$

The remainder of the paper is organized as follows. In Section II, we present the basic protocols examined in this work and in Sections III and IV, we analyze DaF and $\mathrm{AaF}$ strategies, respectively. Finally, conclusions are given in Section VI.

\section{Models AND PROTOCOLS}

We consider a half-duplex dual-hop communication scenario in a cluttered environment depicted in Fig. 1, where the

\footnotetext{
${ }^{3}$ An implementation example can be found in [19].
}

direct path between the source and destination is blocked by an intermediate wall, while relays are located at the periphery of the obstacle (around-the-corner). The relays can communicate with both endpoints (source and destination). During the first hop, the source (without exploiting any CSI) transmits $N / 2$ symbols and the relays listen, while during the second hop, the relays forward a version of the received signal using the same number of symbols. ${ }^{4}$ The channel is assumed to remain constant during the two hops (at least $N$-symbol coherence time) with Rayleigh fading. We further consider a source power constraint

$$
\mathcal{P}_{\text {source }}=\zeta \mathcal{P}_{\text {tot }}
$$

and an aggregate relay power constraint

$$
\mathcal{P}_{\text {relay }}=\sum_{k=1}^{K} \mathcal{P}_{k}=(1-\zeta) \mathcal{P}_{\text {tot }}
$$

where $K$ is the number of relays, $\mathcal{P}_{\text {tot }}$ is the total end-to-end (i.e., source-relay-destination) transmission power, $\mathcal{P}_{\text {source }}$ is the transmission power of the source, $\mathcal{P}_{k}, k=1,2, \ldots, K$, is the transmission power of the $k$ th relay, and $\mathcal{P}_{\text {relay }}$ is the aggregate relay power allocated to the set $\mathcal{S}_{\text {relay }}=\{1,2, \ldots, K\}$ of $K$ relays. Note that if the $k$ th relay does not participate in relaying, $\mathcal{P}_{k}$ is equal to zero. Also, $\zeta \in(0,1]$ and $(1-\zeta) \in[0,1)$ denote the fractions of the total end-to-end power $\mathcal{P}_{\text {tot }}$ allocated to the source transmission and overall relay transmission, respectively.

It should be noted that the optimal power allocation across the source and relays depends on CSI knowledge and can

${ }^{4}$ If the source is allowed to transmit different symbols during the second hop, one channel degree of freedom would not be wasted and the spectral efficiency can be improved [4], [20]. However, in this paper, we are interested in finding the optimal strategy for relay transmissions and hence, simplify their operation. 
be $\mathcal{P}_{\text {source }} \neq \mathcal{P}_{\text {relay }}$ [21]. However, this is feasible only when global CSI about the whole network (including channel states between the relays and destination) is available at the source. In this work, we do not assume CSI at the source. Furthermore, optimal power allocation becomes more important when there is a good link between the source and final destination. None of these conditions are applicable to our study. In fact, our main focus is not just optimal power allocation but a more general question of what relays should do optimally-retransmit or not.

We now provide the model for the received signal in a link $(\mathrm{A} \rightarrow \mathrm{B})$ between two nodes " $\mathrm{A}$ " and "B":

$$
y_{\mathrm{B}}=\alpha_{\mathrm{AB}} x_{\mathrm{A}}+n_{\mathrm{B}}
$$

where $x_{\mathrm{A}}$ is the signal transmitted at the node $\mathrm{A}, \alpha_{\mathrm{AB}} \sim$ $\mathcal{C N}\left(0, \Omega_{\mathrm{AB}}\right)$ is the channel gain between the link $\mathrm{A} \rightarrow \mathrm{B}$, and $n_{\mathrm{B}} \sim \mathcal{C N}\left(0, N_{0}\right)$ is the additive white Gaussian noise (AWGN) at the node B. ${ }^{5}$ For each link, let $\gamma_{\mathrm{AB}} \triangleq\left|\alpha_{\mathrm{AB}}\right|^{2}$ be the instantaneous squared channel strength, which obeys an exponential distribution with hazard rate $1 / \Omega_{\mathrm{AB}}$, denoted by $\gamma_{\mathrm{AB}} \sim \Upsilon\left(1 / \Omega_{\mathrm{AB}}\right)$. The probability density function (p.d.f.) of $\gamma_{\mathrm{AB}}$ is given by

$$
p_{\gamma_{\mathrm{AB}}}(x)=\frac{1}{\Omega_{\mathrm{AB}}} \exp \left(-x / \Omega_{\mathrm{AB}}\right), \quad x \geq 0 .
$$

If the node $\mathrm{A}$ is the source, then $\mathbb{E}\left\{\left|x_{\mathrm{A}}\right|^{2}\right\}=\mathcal{P}_{\text {source }}$. Similarly, if the node A is the $k$ th relay, then $\mathbb{E}\left\{\left|x_{\mathrm{A}}\right|^{2}\right\}=\mathcal{P}_{k}$. Specifically, for each relay $k \in \mathcal{S}_{\text {relay }}$, we designate a link from the source to the $k$ th relay by $\mathrm{S} \rightarrow k$ and a link from the $k$ th relay to the destination by $k \rightarrow \mathrm{D}$. For the links $\mathrm{S} \rightarrow k$ and $k \rightarrow \mathrm{D}$, the received average signal-to-noise ratios (SNRs) are equal to $\eta_{\mathrm{S} k} \triangleq \Omega_{\mathrm{S} k} \mathcal{P}_{\text {source }} / N_{0}$ and $\eta_{k \mathrm{D}} \triangleq \Omega_{k \mathrm{D}} \mathcal{P}_{k} / N_{0}$, respectively. ${ }^{6}$

To reduce overhead and simplify protocol implementation, cooperation is coordinated only every $N$ symbols. As shown in Fig. 1, we consider two modes of coordination: (i) reactive coordination among $\mathrm{DaF}$ relays and (ii) proactive coordination among $\mathrm{DaF}$ or $\mathrm{AaF}$ relays. In a reactive mode, relays that successfully decode the message participate in cooperation, whereas in a proactive mode, specific relays that are selected prior to the source transmission participate in cooperation.

Relay selection can be performed without requiring global CSI at each relay or at a central controller in the network. One possible approach is to use the method of distributed timers proposed in [19], where each relay estimates its own instantaneous channel paths towards the source and the destination. This can be accomplished by listening pilot signals transmitted from the source (Ready-To-Send or RTS) and transmitted from the destination (Clear-to-Send or CTS). Upon receiving CTS, each relay $k$ then starts a timer $T_{k}$ whose duration is inversely

${ }^{5} \mathcal{C N}\left(\mu, \sigma^{2}\right)$ denotes a complex circularly symmetric Gaussian distribution with mean $\mu$ and variance $\sigma^{2}$. Similarly, $\tilde{\mathcal{N}}_{m}(\boldsymbol{\mu}, \boldsymbol{\Sigma})$ denotes a complex $m$ variate Gaussian distribution with a mean vector $\boldsymbol{\mu} \in \mathbb{C}^{m}$ and a covariance matrix $\boldsymbol{\Sigma} \in \mathbb{C}^{m \times m}$. Note that a specific time index is dropped in (3).

${ }^{6} \mathrm{We}$ consider a scenario in which the channel gains for all links are statistically independent. In addition, since we consider a different average channel gain $\mathbb{E}\left\{\gamma_{\mathrm{AB}}\right\}=\Omega_{\mathrm{AB}}$ for each link, the noise variances at all nodes are normalized to $N_{0}$ without loss of generality. Throughout the paper, we use the term 'SNR' to refer to instantaneous SNR. The term 'average SNR' is explicitly used to describe the SNR averaged over the fading ensemble.

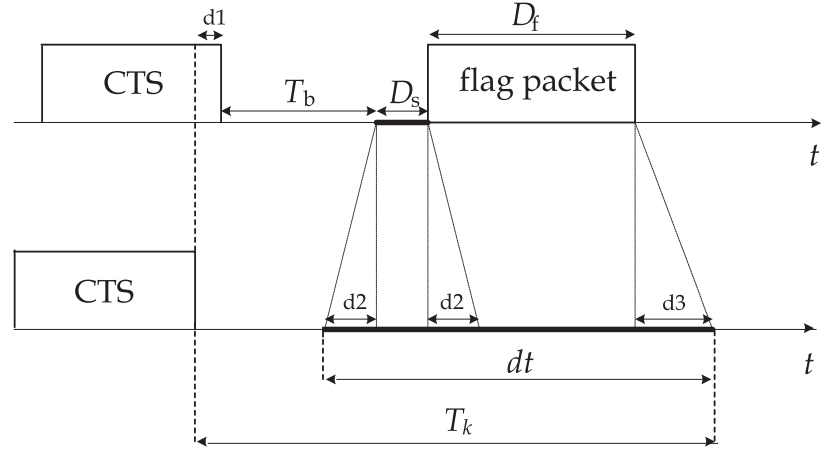

Fig. 2. Distributed relay selection: Upon reception of CTS from destination, each relay starts its own timer $T_{k}$ with a metric of its own channel conditions. The relay with the highest metric will have its timer $T_{b}$ expire first and broadcast a flag packet, notifying its availability for relaying to the rest of the terminals.

proportional to a metric depending only on its own channel gains towards source $\left|\alpha_{S k}\right|$ and destination $\left|\alpha_{k \mathrm{D}}\right|$ (see Fig. 2). The timer $T_{\mathrm{b}}$ of the "best" relay $b$ expires first and a flag packet with duration $D_{\mathrm{f}}$ notifies the rest of the network about its availability. ${ }^{7}$

Since all cooperative relays compete to access the wireless medium according to their own channel conditions, there is a finite probability that any two relays have their timer expire within the same time interval $d t$ and transmit their flag packets (Fig. 2). In that case, the destination can assess that more than one relays are possible candidates. Such probability depends on 1) the propagation delays $d 1, d 3$ from destination to relays, ${ }^{8}$ 2) propagation delays $d 2$ between the relays, 3) radio listen-totransmit switch time $D_{\mathrm{s}}$ and 4) duration $D_{\mathrm{f}}$ of the flag packet when relays cannot listen to each other (the case of hidden relay terminals). That probability was analytically calculated for any type of wireless fading statistics in [19] and [22]. It was shown that opportunistic relay selection can be completed within a fraction of the channel coherence time. Additional details regarding the above distributed relay selection protocol without global CSI and implementation examples with lowcost radios can be found in [19].

\section{DECODE-AND-ForWARD RELAYING}

\section{A. Reactive DaF}

1) Reactive Multiple-Relay DaF: In a reactive MR scheme with $\mathrm{DaF}$ strategy, the relays that successfully receive the message during the first phase regenerate and transmit it during the second phase, possibly through a distributed spacetime code [7]. The transmissions during the second phase are performed only by a subset $\mathcal{D}$ of $K$ relays, defined by

$$
\mathcal{D} \triangleq\left\{k \in \mathcal{S}_{\text {relay }}: \frac{1}{2} \log _{2}\left(1+\zeta \gamma_{\mathrm{S} k} \frac{\mathcal{P}_{\text {tot }}}{N_{0}}\right) \geq R\right\}
$$

where $R$ denotes the end-to-end spectral efficiency in bps/Hz. In (5), the decoding at relay $k$ is assumed to be successful if $\frac{1}{2} \log _{2}\left(1+\zeta \gamma_{S k} \mathcal{P}_{\text {tot }} / N_{0}\right) \geq R$, i.e., no outage event happens

\footnotetext{
${ }^{7}$ Note that no explicit time-synchronization protocol is required among the relays.

${ }^{8} d 1$ is one-way propagation delay and $d 3$ includes round-trip propagation delay.
} 
during the first phase [3], [7]. Since communication happens in two half-duplex hops, the required spectral efficiency per hop is equal to $2 R$ so that the end-to-end spectral efficiency is $R$, which is comparable to direct non-cooperative communication.

Let $\mathcal{D}_{\ell} \subseteq \mathcal{S}_{\text {relay }}$ be a decoding subset with $\ell$ relays (i.e., cardinality $\left|\mathcal{D}_{\ell}\right|=\ell$ ). Then, we have

$$
\begin{aligned}
\operatorname{Pr}\left\{\mathcal{D}_{\ell}\right\} & =\prod_{i \in \mathcal{D}_{\ell}} \operatorname{Pr}\left\{\gamma_{\mathrm{S} i} \geq \kappa_{1}\right\} \prod_{j \notin \mathcal{D}_{\ell}} \operatorname{Pr}\left\{\gamma_{\mathrm{S}_{j}} \leq \kappa_{1}\right\} \\
& =\prod_{i \in \mathcal{D}_{\ell}} e^{-\kappa_{1} / \Omega_{\mathrm{S} i}} \prod_{j \notin \mathcal{D}_{\ell}}\left(1-e^{-\kappa_{1} / \Omega_{\mathrm{S} j}}\right)
\end{aligned}
$$

where $\kappa_{1}=\frac{2^{2 R}-1}{\zeta \mathcal{P}_{\text {tot }} / N_{0}}$. The outage probability for reactive MR transmissions with $\mathrm{DaF}$ strategy can be written as

$$
P_{\text {MR-DaF }}^{(\text {react })}(\text { outage })=\sum_{\ell=0}^{K} \sum_{\mathcal{D}_{\ell}} \operatorname{Pr}\left\{\text { outage } \mid \mathcal{D}_{\ell}\right\} \operatorname{Pr}\left\{\mathcal{D}_{\ell}\right\}
$$

where the second summation is over all $\left(\begin{array}{c}K \\ \ell\end{array}\right)$ different decoding subsets with exactly $\ell$ successfully decodable relays. ${ }^{9}$ In (7), the conditional outage probability is given by

$$
\operatorname{Pr}\left\{\text { outage } \mid \mathcal{D}_{\ell}\right\}=\operatorname{Pr}\left\{\frac{1}{2} \log _{2}\left(1+\sum_{k \in \mathcal{D}_{\ell}} \gamma_{k \mathrm{D}} \frac{\mathcal{P}_{k}}{N_{0}}\right)<R\right\}
$$

with $\sum_{k \in \mathcal{D}_{\ell}} \mathcal{P}_{k}=\mathcal{P}_{\text {relay }}$.

$$
\begin{aligned}
\text { Let } & \left\{\varphi_{i}\left(\mathcal{D}_{\ell}\right)\right\}_{i=1}^{\ell}=\left\{\eta_{k \mathrm{D}}\right\}_{k \in \mathcal{D}_{\ell}} \text { and } \\
& \boldsymbol{A}\left(\mathcal{D}_{\ell}\right)=\operatorname{diag}\left(\varphi_{1}\left(\mathcal{D}_{\ell}\right), \varphi_{2}\left(\mathcal{D}_{\ell}\right), \ldots, \varphi_{\ell}\left(\mathcal{D}_{\ell}\right)\right) .
\end{aligned}
$$

Then, using Theorem 2 in Appendix A, we obtain the conditional outage probability $\operatorname{Pr}\left\{\right.$ outage $\left.\mid \mathcal{D}_{\ell}\right\}$ as (9), shown at the bottom of the page, where $\varrho\left(\mathcal{A}\left(\mathcal{D}_{\ell}\right)\right)$ is the number of distinct diagonal elements of $\boldsymbol{A}\left(\mathcal{D}_{\ell}\right), \varphi_{\langle 1\rangle}\left(\mathcal{D}_{\ell}\right)>\varphi_{\langle 2\rangle}\left(\mathcal{D}_{\ell}\right)>$ $\ldots>\varphi_{\left\langle\varrho\left(\mathcal{A}\left(\mathcal{D}_{\ell}\right)\right)\right\rangle}\left(\mathcal{D}_{\ell}\right)$ are the distinct diagonal elements in decreasing order, $\tau_{i}\left(\boldsymbol{A}\left(\mathcal{D}_{\ell}\right)\right)$ is the multiplicity of $\varphi_{\langle i\rangle}\left(\mathcal{D}_{\ell}\right)$, and $\mathcal{X}_{i, j}\left(\boldsymbol{A}\left(\mathcal{D}_{\ell}\right)\right)$ is the $(i, j)$ th characteristic coefficient of $\mathcal{A}\left(\mathcal{D}_{\ell}\right)$ [23]. Combining (6), (7), and (9) gives (10), shown at the bottom of the page.

\footnotetext{
${ }^{9}$ The equality in (7) is due to the total probability theorem over disjoint sets $\mathcal{D}_{\ell}$ that partition the sample space. Note that there are $2^{K}$ possible decoding subsets for $K$ relays, including $\mathcal{D}_{0}$, i.e., the set with no decodable relay during the first hop of the protocol.
}

2) Reactive Opportunistic DaF: The following theorem establishes the fact that opportunistic DaF relaying is outageoptimal, that is, it is equivalent in outage behavior to the optimal DaF strategy that employs all potential relays.

Theorem 1: For a reactive DaF relaying scheme with the aggregate power constraint (2), choosing the "best" relay $b_{\text {react-DaF }}^{*}$ that maximizes the instantaneous channel strength between the links $k \rightarrow \mathrm{D}$ for all $k \in \mathcal{D}_{\ell}$, that is,

$$
b_{\text {react-DaF }}^{*}=\arg \max _{k \in \mathcal{D}_{\ell}} \gamma_{k \mathrm{D}}
$$

is outage-optimal.

Proof: For any reactive $\mathrm{DaF}$ relaying scheme with the aggregate power constraint (2), the received SNR at the destination is upper bounded as

$$
\begin{aligned}
\sum_{k \in \mathcal{D}_{\ell}} \gamma_{k \mathrm{D}} \frac{\mathcal{P}_{k}}{N_{0}} & \leq \sum_{k \in \mathcal{D}_{\ell}}\left(\max _{k \in \mathcal{D}_{\ell}} \gamma_{k \mathrm{D}}\right) \frac{\mathcal{P}_{k}}{N_{0}} \\
& =(1-\zeta) \max _{k \in \mathcal{D}_{\ell}} \gamma_{k \mathrm{D}} \frac{\mathcal{P}_{\text {tot }}}{N_{0}} .
\end{aligned}
$$

Therefore, the conditional outage probability in (8) for any reactive DaF relaying scheme is lower bounded as follows:

$$
\begin{aligned}
& \operatorname{Pr}\left\{\text { outage } \mid \mathcal{D}_{\ell}\right\} \\
& \geq \operatorname{Pr}\left\{\frac{1}{2} \log _{2}\left(1+(1-\zeta) \max _{k \in \mathcal{D}_{\ell}} \gamma_{k \mathrm{D}} \frac{\mathcal{P}_{\text {tot }}}{N_{0}}\right)<R\right\} \\
& =\prod_{k \in \mathcal{D}_{\ell}} \operatorname{Pr}\left\{\gamma_{k \mathrm{D}}<\kappa_{2}\right\}
\end{aligned}
$$

where $\kappa_{2}=\frac{2^{2 R}-1}{(1-\zeta) \mathcal{P}_{\text {tot }} / N_{0}}$. Since the maximum received SNR in (12) and the minimum conditional outage probability in the right-hand side of (13) are achieved by the single opportunistic relay-selection rule (11), we complete the proof of its outage optimality.

Note that (14) states simply that if the "best" relay fails, then all relays in $\mathcal{D}_{\ell}$ fail because the "best" relay has the strongest path $\gamma_{b_{\text {react-DaF }}^{*} \mathrm{D}}$ between the links $k \rightarrow \mathrm{D}$ for all $k \in$ $\mathcal{D}_{\ell}$. We remark that the minimization of (14) holds for any power allocation $\zeta$. For quasi-static fading environments, a simple method can be devised to select the relay with the maximum channel strength $\gamma_{b_{\text {react }}^{*} \mathrm{DF}} \mathrm{D}$ in a distributed manner similar to the work in [19] and [22].

$$
\operatorname{Pr}\left\{\text { outage } \mid \mathcal{D}_{\ell}\right\}=1-\sum_{i=1}^{\varrho\left(\mathcal{A}\left(\mathcal{D}_{\ell}\right)\right)} \sum_{j=1}^{\tau_{i}\left(\mathcal{A}\left(\mathcal{D}_{\ell}\right)\right)} \sum_{k=0}^{j-1}\left[\frac{\mathcal{X}_{i, j}\left(\mathcal{A}\left(\mathcal{D}_{\ell}\right)\right)}{k !}\left(\frac{2^{2 R}-1}{\varphi_{\langle i\rangle}\left(\mathcal{D}_{\ell}\right)}\right)^{k} \exp \left(-\frac{2^{2 R}-1}{\varphi_{\langle i\rangle}\left(\mathcal{D}_{\ell}\right)}\right)\right]
$$

$$
\begin{aligned}
&\left.P_{\mathrm{MR}-\mathrm{DaF}}^{\text {(react) }} \text { (outage) }\right)=\sum_{\ell=0}^{K} \sum_{\mathcal{D}_{\ell}} {\left[\prod_{i \in \mathcal{D}_{\ell}} e^{-\kappa_{1} / \Omega_{\mathrm{S} i}} \prod_{j \notin \mathcal{D}_{\ell}}\left(1-e^{-\kappa_{1} / \Omega_{\mathrm{S} j}}\right)\right.} \\
&\left.\times\left\{1-\sum_{i=1}^{\varrho\left(\mathcal{A}\left(\mathcal{D}_{\ell}\right)\right)} \sum_{j=1}^{\tau_{i}\left(\mathcal{A}\left(\mathcal{D}_{\ell}\right)\right)} \sum_{k=0}^{j-1} \frac{\mathcal{X}_{i, j}\left(\mathcal{A}\left(\mathcal{D}_{\ell}\right)\right)}{k !}\left(\frac{2^{2 R}-1}{\varphi_{\langle i\rangle}\left(\mathcal{D}_{\ell}\right)}\right)^{k} \exp \left(-\frac{2^{2 R}-1}{\varphi_{\langle i\rangle}\left(\mathcal{D}_{\ell}\right)}\right)\right\}\right]
\end{aligned}
$$


Using (6) and (7) in conjunction with (14) for the opportunistic relay-selection rule (11), we obtain the outage probability for reactive opportunistic $\mathrm{DaF}$ relaying as

$$
\begin{aligned}
& P_{\text {Opp-DaF }}^{\text {(react) }} \text { (outage) } \\
& =\sum_{\ell=0}^{K} \sum_{\mathcal{D}_{\ell}}\left[\prod_{i \in \mathcal{D}_{\ell}} e^{-\frac{\kappa_{1}}{\Omega_{\mathrm{Si}}}}\left(1-e^{-\frac{\kappa_{2}}{\Omega_{i \mathrm{D}}}}\right) \prod_{j \notin \mathcal{D}_{\ell}}\left(1-e^{-\frac{\kappa_{1}}{\Omega_{\mathrm{S} j}}}\right)\right] \\
& =\prod_{k=1}^{K}\left[1-\exp \left\{-\frac{2^{2 R}-1}{\mathcal{P}_{\text {tot }} / N_{0}}\left(\frac{1}{\zeta \Omega_{\mathrm{S} k}}+\frac{1}{(1-\zeta) \Omega_{k \mathrm{D}}}\right)\right\}\right]
\end{aligned}
$$

where the last equality follows from the multinomial equality

$$
\begin{aligned}
& \prod_{k=1}^{K}\left(1-a_{k} b_{k}\right)=\prod_{k=1}^{K}\left[a_{k}\left(1-b_{k}\right)+\left(1-a_{k}\right)\right] \\
& =\sum_{\ell=0}^{K} \sum_{\mathcal{S}_{\ell} \subseteq\{1, \ldots, K\}}\left[\prod_{i \in \mathcal{S}_{\ell}\left|\mathcal{S}_{\ell}\right|=\ell} a_{i}\left(1-b_{i}\right) \prod_{j \notin \mathcal{S}_{\ell}}\left(1-a_{j}\right)\right] .
\end{aligned}
$$

Note that (15) implies that opportunistic DaF relaying is in outage only when all of the relays are in outage. In this case, no other schemes can communicate reliably at rate $R$. Hence, the reactive opportunistic DaF relaying is optimal under the aggregate relay power constraint (2) in a sense that it minimizes the end-to-end outage probability.

In contrast to our single-relay opportunistic rule, one may consider selection of the relay that maximizes the average forward channel gain among the decoding set (see, e.g., [24], [25]):

$$
\begin{aligned}
b_{\text {react-DaF }}^{*} & =\arg \max _{k \in \mathcal{D}_{\ell}} \mathbb{E}\left\{\gamma_{k \mathrm{D}}\right\} \\
& =\arg \max _{k \in \mathcal{D}_{\ell}} \Omega_{k \mathrm{D}} .
\end{aligned}
$$

\section{B. Proactive DaF}

It might seem that selecting a single relay before information is transmitted from the source, could potentially result in degraded performance. On the other hand, selecting a single relay for information forwarding simplifies the receiver design and the overall network operation, since proactive selection is equivalent to routing. In what follows, we show that such choice on protocol design incurs no performance loss.

1) Proactive Opportunistic DaF: In proactive opportunistic relaying, the "best" relay $b_{\text {proact-DaF }}^{*}$ is chosen prior to the source transmission among a collection of $K$ possible candidates in a distributed fashion that requires each relay to know its own instantaneous signal strength (but not phase) between the links $\mathrm{S} \rightarrow k$ and $k \rightarrow \mathrm{D}$, for each relay $k \in \mathcal{S}_{\text {relay }} .{ }^{10}$ The "best" relay $b_{\text {proact-DaF }}^{*}$ is chosen to maximize the minimum of the weighted channel strengths between the links $\mathrm{S} \rightarrow k$ and $k \rightarrow \mathrm{D}$ for all $k \in \mathcal{S}_{\text {relay }}:{ }^{11}$

$$
b_{\text {proact-DaF }}^{*}=\arg \max _{k \in \mathcal{S}_{\text {relay }}} W_{k}^{(\mathrm{DaF})}
$$

\footnotetext{
${ }^{10}$ Relay selection can be accomplished using a method of distributed timers described in Section II, without requiring global CSI.

${ }^{11}$ Instead of the minimum, the harmonic mean of two path strengths has been also considered in [19].
}

where $W_{k}^{(\mathrm{DaF})}=\min \left\{\zeta \gamma_{\mathrm{S} k},(1-\zeta) \gamma_{k \mathrm{D}}\right\}$.

In this case, communication through the "best" opportunistic relay fails due to outage when either of the two hops (from the source to the best relay or from the best relay to destination) fail. Recall that

$$
W_{k}^{(\mathrm{DaF})} \sim \Upsilon\left(\frac{1}{\zeta \Omega_{\mathrm{S} k}}+\frac{1}{(1-\zeta) \Omega_{k \mathrm{D}}}\right)
$$

which follows from the fact that the minimum of two independent exponential random variables (r.v.'s) is again an exponential r.v. with a hazard rate equal to the sum of the two hazard rates. From (18) and (19), we obtain the outage probability for proactive opportunistic DaF relaying as follows:

$$
\begin{aligned}
& P_{\mathrm{Opp}-\mathrm{DaF}}^{\text {proact }}(\text { outage }) \\
& =\operatorname{Pr}\left\{W_{b_{\text {proact-DaF }}^{*}}^{(\text {DaF) }}<\frac{2^{2 R}-1}{\mathcal{P}_{\text {tot }} / N_{0}}\right\} \\
& =\operatorname{Pr}\left\{\max _{k \in \mathcal{S}_{\text {relay }}} W_{k}^{(\mathrm{DaF})}<\frac{2^{2 R}-1}{\mathcal{P}_{\text {tot }} / N_{0}}\right\} \\
& =\prod_{k=1}^{K} \operatorname{Pr}\left\{W_{k}^{(\mathrm{DaF})}<\frac{2^{2 R}-1}{\mathcal{P}_{\text {tot }} / N_{0}}\right\} \\
& =\prod_{k=1}^{K}\left[1-\exp \left\{-\frac{2^{2 R}-1}{\mathcal{P}_{\mathrm{tot}} / N_{0}}\left(\frac{1}{\zeta \Omega_{\mathrm{S} k}}+\frac{1}{(1-\zeta) \Omega_{k \mathrm{D}}}\right)\right\}\right] .
\end{aligned}
$$

It is worth remarking that the outage probability in (20) agrees exactly with that in (15) for reactive opportunistic relays with $\mathrm{DaF}$ strategy. Since we have shown in the previous section that reactive opportunistic relaying is outage-optimal, the proactive opportunistic "max-min" relay selection in (18) is also outage-optimal. Moreover, proactive coordination requires a smaller cooperation overhead in reception energy since all relays, except a single opportunistic relay, can enter an idle mode during the first hop of the protocol. Therefore, our proactive strategy can be viewed as energy-efficient routing in the network. In contrast, the reactive schemes require all relays to receive information during the first hop and therefore, cooperation overhead in reception energy scales proportionally with the network size.

\section{AMPLIFY-AND-FORWARD RELAYING}

For the case of AaF, each relay normalizes the signal $y_{k}, k \in$ $\mathcal{S}_{\text {relay }}$, received during the first phase of the protocol (Fig. 1) and transmits $x_{k}=\sqrt{\mathcal{P}_{k}} \frac{y_{k}}{\sqrt{\mathbb{E}\left\{\left|y_{k}\right|^{2}\right\}}}$ during the second phase of the protocol. The received signal $y_{\mathrm{D}}$ at the destination after the second phase of the protocol can be written as

$$
y_{\mathrm{D}}=h x_{\mathrm{S}}+n_{\mathrm{D}}
$$

where

$$
h=\frac{1}{\check{\sigma}} \sum_{k=1}^{K} \sqrt{\frac{\mathcal{P}_{k}}{\Omega_{\mathrm{S} k} \mathcal{P}_{\text {source }}+N_{0}}} \alpha_{\mathrm{S} k} \alpha_{k \mathrm{D}}
$$

with

$$
\check{\sigma}^{2}=1+\sum_{k=1}^{K} \frac{\mathcal{P}_{k}\left|\alpha_{k \mathrm{D}}\right|^{2}}{\Omega_{\mathrm{S} k} \mathcal{P}_{\text {source }}+N_{0}} .
$$


When conditioned on the channel gains from relays to the destination $\left\{\alpha_{k \mathrm{D}}\right\}_{k=1}^{K}$, the noise parameter $n_{\mathrm{D}}$ at the destination is found to be a zero-mean complex Gaussian random variable with variance $N_{0}$. From (21), it is easy to find that the mutual information for the AaF strategy with $K$ relays subject to the power constraint (2) is given by

$$
\begin{aligned}
\mathcal{I}_{\mathrm{MR}-\mathrm{AaF}} & =\frac{1}{2} \log _{2}\left(1+|h|^{2} \frac{\mathcal{P}_{\text {source }}}{N_{0}}\right) \\
= & \frac{1}{2} \log _{2}\left\{1+\frac{\left|\sum_{k=1}^{K} \sqrt{\frac{\mathcal{P}_{k}}{\Omega_{\mathrm{Sk}} \mathcal{P}_{\text {source }}+N_{0}}} \alpha_{\mathrm{S} k} \alpha_{k \mathrm{D}}\right|^{2}}{\left(1+\sum_{k=1}^{K} \frac{\mathcal{P}_{k}\left|\alpha_{k \mathrm{D}}\right|^{2}}{\Omega_{\mathrm{S} k} \mathcal{P}_{\text {source }}+N_{0}}\right)} \frac{\mathcal{P}_{\text {source }}}{N_{0}}\right\} .
\end{aligned}
$$

\section{A. Opportunistic AaF}

From (24), we see that the maximum mutual information with a single-relay selection, i.e., the mutual information for opportunistic AaF relaying is

$\mathcal{I}_{\text {Opp-AaF }}=\max _{k \in \mathcal{S}_{\text {relay }}} \frac{1}{2} \log _{2}\left\{1+\frac{\gamma_{\mathrm{S} k} \gamma_{k \mathrm{D}}}{\frac{\zeta}{1-\zeta} \Omega_{\mathrm{S} k}+\frac{N_{0}}{\mathcal{P}_{\text {relay }}}+\gamma_{k \mathrm{D}}} \frac{\mathcal{P}_{\text {source }}}{N_{0}}\right\}$.

Hence, for opportunistic relaying, the "best" relay $b_{\mathrm{AaF}}^{*}$ among $K$ relays in $\mathcal{S}_{\text {relay }}$ is chosen proactively to maximize the mutual information (or to minimize the outage probability) as follows:

$$
b_{\mathrm{AaF}}^{*}=\arg \max _{k \in \mathcal{S}_{\text {relay }}} W_{k}^{(\mathrm{AaF})}
$$

where

$$
W_{k}^{(\mathrm{AaF})}=\frac{\gamma_{\mathrm{S} k} \gamma_{k \mathrm{D}}}{\frac{\zeta}{1-\zeta}\left(1+\frac{1}{\eta_{\mathrm{Sk} k}}\right) \Omega_{\mathrm{S} k}+\gamma_{k \mathrm{D}}} .
$$

Note that individual relays do not need to acquire CSI about the links of other relays and hence, the opportunistic relay (26) can also be selected in a distributed manner [19], [22].

Then, using Theorem 3 in Appendix B, we obtain the outage probability for opportunistic $\mathrm{AaF}$ relaying as (28), shown at the bottom of the page.

\section{Numerical AND Simulation Results}

In this section, we give numerical examples of the outage probability as a function of $\mathcal{P}_{\text {tot }} / N_{0}$ with power allocation $\zeta=0.5$. The optimal power allocation $\zeta$ is feasible, only when the source has knowledge of the overall network topology in terms of the average channel gains $\Omega_{\mathrm{S} k}$ and $\Omega_{k \mathrm{D}}$ for all participating relays $k \in \mathcal{S}_{\text {relay }}$. However, this is impractical since

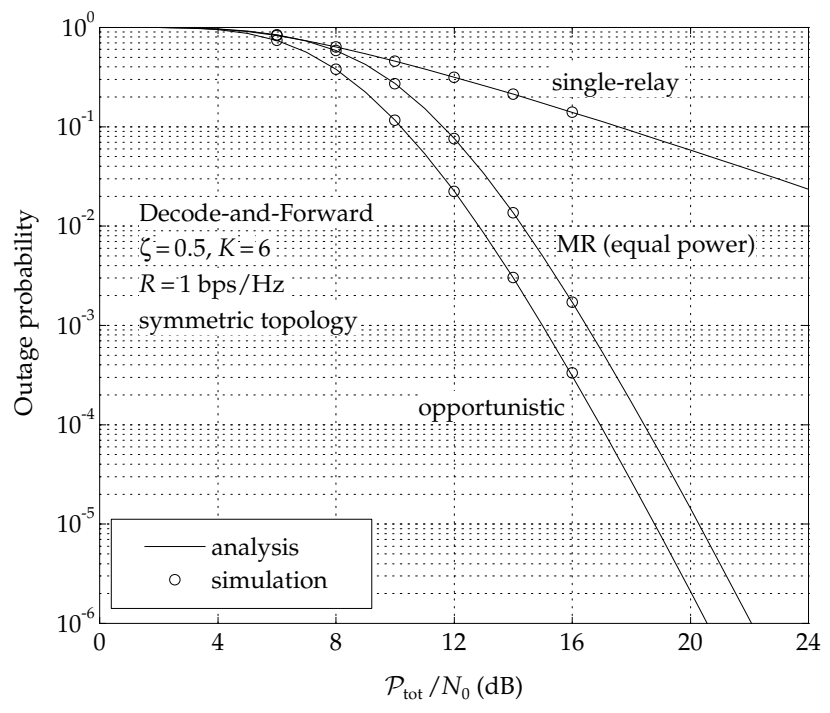

Fig. 3. Outage probability as a function of $\mathcal{P}_{\text {tot }} / N_{0}$ for the DaF strategy at the end-to-end spectral efficiency $R=1 \mathrm{bps} / \mathrm{Hz}$ in symmetric channels. $\zeta=0.5, K=6$, and $\Omega_{\mathrm{S} k}=\Omega_{k \mathrm{D}}=1, k=1,2, \ldots, 6$. Opportunistic relaying is compared with reactive equal-power MR transmissions and singlerelay selection based on the maximum average channel gain $\max _{k \in \mathcal{D}_{\ell}} \Omega_{k \mathrm{D}}$.

such knowledge requires considerable overhead. Therefore, the equal-power allocation to the source and the opportunistic relay, i.e., $\zeta=0.5$ is a natural choice. We further quantify the performance difference between $\zeta=0.5$ and optimal choice of $\zeta$ that requires global CSI at the transmitter and the relay. Our results accommodate both symmetric and asymmetric topologies for $\mathrm{DaF}$ or $\mathrm{AaF}$ strategy.

\section{A. Decode-and-Forward Relaying}

Fig. 3 shows the outage probability as a function of $\mathcal{P}_{\text {tot }} / N_{0}$ for the $\mathrm{DaF}$ strategy with 6 relays $(K=6)$ at the end-to-end spectral efficiency $R=1 \mathrm{bps} / \mathrm{Hz}$ in symmetric channels with $\Omega_{\mathrm{S} k}=\Omega_{k \mathrm{D}}=1, k=1,2, \ldots, 6$. In this figure, we show the performance of (i) proactive opportunistic $\mathrm{DaF}$ relaying, (ii) reactive $\mathrm{DaF}$ relaying with equal-power MR transmissions, and (iii) reactive $\mathrm{DaF}$ relaying via single-relay selection based on the maximum average channel gain $\max _{k \in \mathcal{D}_{\ell}} \Omega_{k \mathrm{D}}$. Fig. 4 compares the same scenarios in asymmetric channels with $\left\{\Omega_{\mathrm{S} k}\right\}_{k=1}^{K}=\left\{\Omega_{k \mathrm{D}}\right\}_{k=1}^{K}=\{4.5,0.5,0.4,0.3,0.2,0.1\}$. Note that for the symmetric case, single-relay selection based on the average channel gains amounts to selecting just one successful relay randomly (since all relays in the decoding subset $\mathcal{D}_{\ell}$ have the same average channel gain to the destination) and transmitting with full relaying power $\mathcal{P}_{\text {relay }}$. Note also that under limited channel knowledge at each relay, the optimal

$$
\begin{aligned}
P_{\text {Opp-AaF }}(\text { outage }) & =\operatorname{Pr}\left\{W_{b_{\mathrm{AaF}}^{*}}^{(\mathrm{AaF})}<\kappa_{1}\right\} \\
& =\operatorname{Pr}\left\{\max _{k \in \mathcal{S}_{\text {relay }}} W_{k}^{(\mathrm{AaF})}<\kappa_{1}\right\} \\
& =\prod_{k=1}^{K}\left[1-\frac{1}{\Omega_{k \mathrm{D}}} \int_{0}^{\infty} \exp \left\{-\frac{2^{2 R}-1}{\eta_{\mathrm{S} k}}\left(1+\frac{\zeta}{1-\zeta}\left(1+\frac{1}{\eta_{\mathrm{S} k}}\right) \frac{\Omega_{\mathrm{S} k}}{z}\right)-\frac{z}{\Omega_{k \mathrm{D}}}\right\} d z\right] .
\end{aligned}
$$




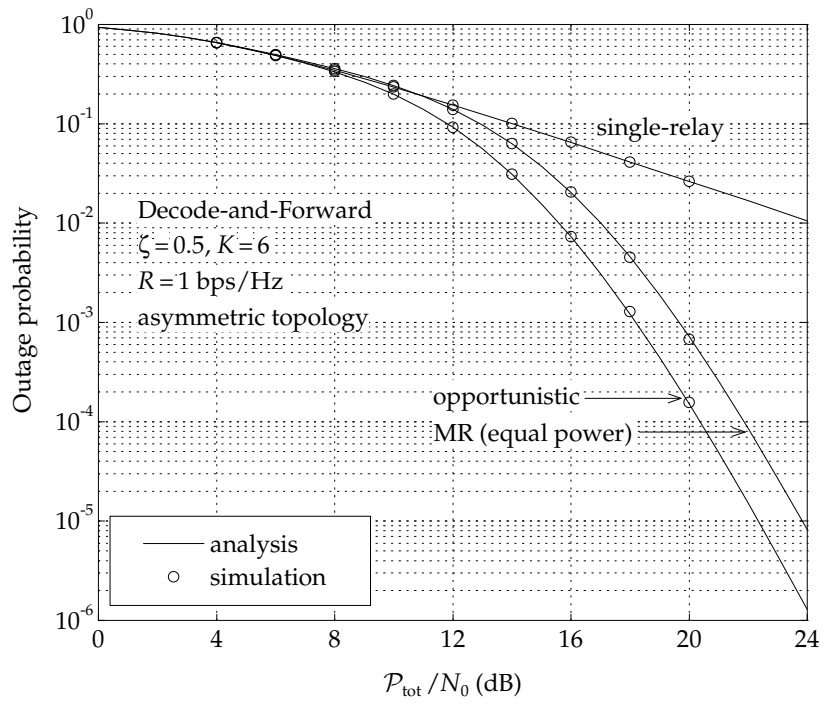

Fig. 4. Outage probability as a function of $\mathcal{P}_{\text {tot }} / N_{0}$ for the DaF strategy at the end-to-end spectral efficiency $R=1 \mathrm{bps} / \mathrm{Hz}$ in asymmetric channels. $\zeta=$ $0.5, K=6$, and $\left\{\Omega_{\mathrm{S} k}\right\}_{k=1}^{K}=\left\{\Omega_{k \mathrm{D}}\right\}_{k=1}^{K}=\{4.5,0.5,0.4,0.3,0.2,0.1\}$. Opportunistic relaying is compared with reactive equal-power MR transmissions and single-relay selection based on the maximum average channel gain $\max _{k \in \mathcal{D}_{\ell}} \Omega_{k \mathrm{D}}$.

power allocation for MR transmission with DaF strategy is infeasible and equal power for the decoding subset $\mathcal{D}_{\ell}$, i.e., $\mathcal{P}_{k}=\mathcal{P}_{\text {relay }} / \ell$ for all $k \in \mathcal{D}_{\ell}$ is a reasonable solution in reactive $\mathrm{DaF}$ relaying.

Both figures show that opportunistic relaying, despite its simplicity, provides a gain in $\mathcal{P}_{\text {tot }} / N_{0}$ on the order of $2 \mathrm{~dB}$ relative to MR transmission with $\mathrm{DaF}$ strategy. This finding reveals that cooperative diversity gains do not necessarily arise from simultaneous transmissions but instead, resilience to fading arises from the availability of several potential paths towards the destination. It is therefore beneficial to select the "best" one. ${ }^{12}$ In contrast to single opportunistic relay selection, Figs. 3 and 4 also show that single-relay selection based on average channel gains incurs a substantial penalty loss. This is due to the fact that selecting a relay with average channel gains removes potential selection diversity benefits.

Fig. 5 and Fig. 6 show the performance of opportunistic DaF relaying as a function of $\zeta$ for the symmetric and asymmetric scenarios, respectively. It is shown that $\zeta=0.5$ is optimal. This can be verified analytically by differentiating (15) or (20). We note however that for the general case of $\Omega_{\mathrm{S} k} \neq \Omega_{k \mathrm{D}}$, for any relay $k$, optimal $\zeta^{*}$ will be different from 0.5 .

\section{B. Amplify-and-Forward Relaying}

The outage probability as a function of $\mathcal{P}_{\text {tot }} / N_{0}$ for the $\mathrm{AaF}$ strategy with 6 relays $(K=6)$ at the end-to-end spectral efficiency $R=1 \mathrm{bps} / \mathrm{Hz}$ is plotted in Figs. 7 and 8 for the symmetric channels $\left(\Omega_{\mathrm{S} k}=\Omega_{k \mathrm{D}}=1\right.$, $k=1,2, \ldots, 6)$ and asymmetric channels $\left(\left\{\Omega_{\mathrm{S} k}\right\}_{k=1}^{K}=\right.$ $\left.\left\{\Omega_{k \mathrm{D}}\right\}_{k=1}^{K}=\{4.5,0.5,0.4,0.3,0.2,0.1\}\right)$, respectively. We

\footnotetext{
${ }^{12}$ The main difficulty here is to have the network as a whole entity cooperate in order to rapidly discover the best path with minimal overhead. Ideas on how such selection can be performed in a distributed manner for slow fading environments can be found in [19], where actual implementations with lowcost radios were demonstrated.
}

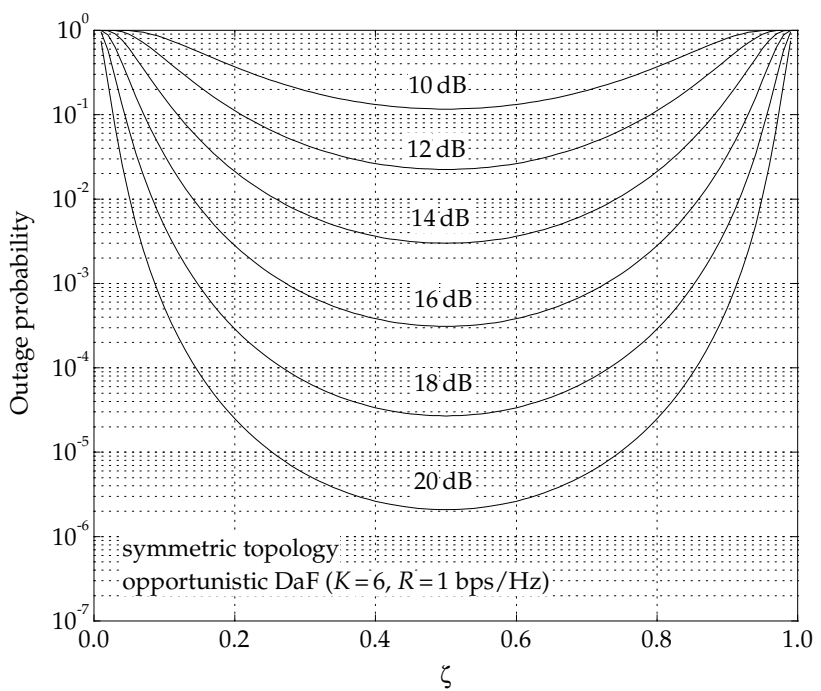

Fig. 5. Outage probability as a function of power allocation $\zeta$ for opportunistic DaF relaying at the end-to-end spectral efficiency $R=1 \mathrm{bps} / \mathrm{Hz}$ in symmetric channels when $\mathcal{P}_{\text {tot }} / N_{0}=10,12,14,16,18$, and $20 \mathrm{~dB}$. $K=6$ and $\Omega_{\mathrm{S} k}=\Omega_{k \mathrm{D}}=1, k=1,2, \ldots, 6$.

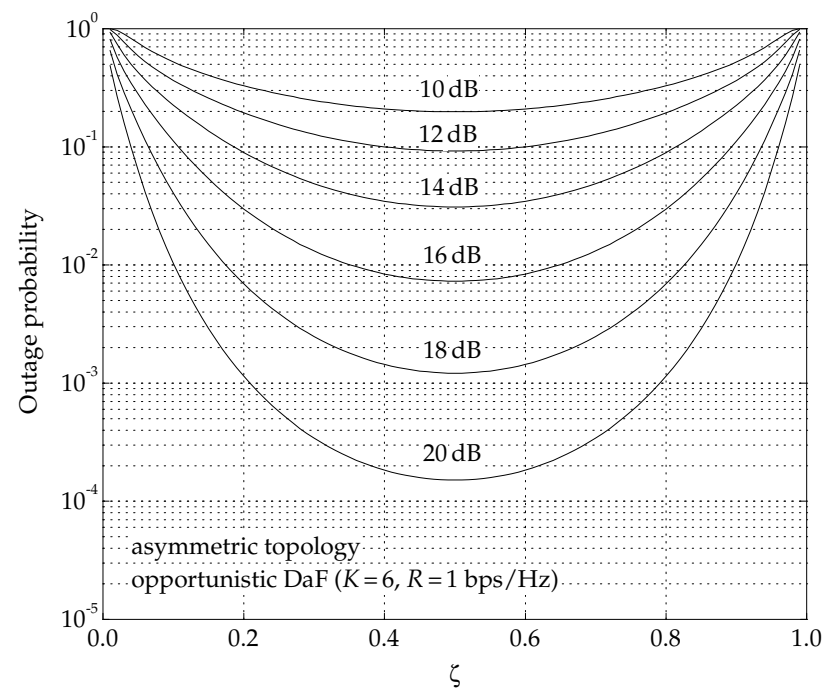

Fig. 6. Outage probability as a function of power allocation $\zeta$ for opportunistic $\mathrm{DaF}$ relaying at the end-to-end spectral efficiency $R=1 \mathrm{bps} / \mathrm{Hz}$ in asymmetric channels when $\mathcal{P}_{\text {tot }} / N_{0}=10,12,14,16,18$, and $20 \mathrm{~dB}$. $K=6$ and $\left\{\Omega_{\mathrm{S} k}\right\}_{k=1}^{K}=\left\{\Omega_{k \mathrm{D}}\right\}_{k=1}^{K}=\{4.5,0.5,0.4,0.3,0.2,0.1\}$.

compare the performance of (i) opportunistic AaF relaying, (ii) AaF relaying with equal-power MR transmissions, and (iii) $\mathrm{AaF}$ relaying with single-relay selection based on the maximum average channel gain $\max _{k \in \mathcal{S}_{\text {relay }}} \Omega_{\mathrm{S} k}$ (or $\left.\max _{k \in \mathcal{S}_{\text {relay }}} \Omega_{k \mathrm{D}}\right)$. Note that for the symmetric case, singlerelay selection based on the average channel gains amounts to selecting just one relay randomly (since all relays have the same average channel gains towards the source and destination) and transmitting with full relaying power $\mathcal{P}_{\text {relay }}$. For the asymmetric case studied, single-relay selection based on the average channel gains amounts to selecting the relay with $\Omega_{\mathrm{S} k}=\Omega_{k \mathrm{D}}=4.5$.

Both figures reveal significant gains of opportunistic $\mathrm{AaF}$ relaying compared to MR transmission with AaF strategy. This is true even for the symmetric scenario, where all relays have 


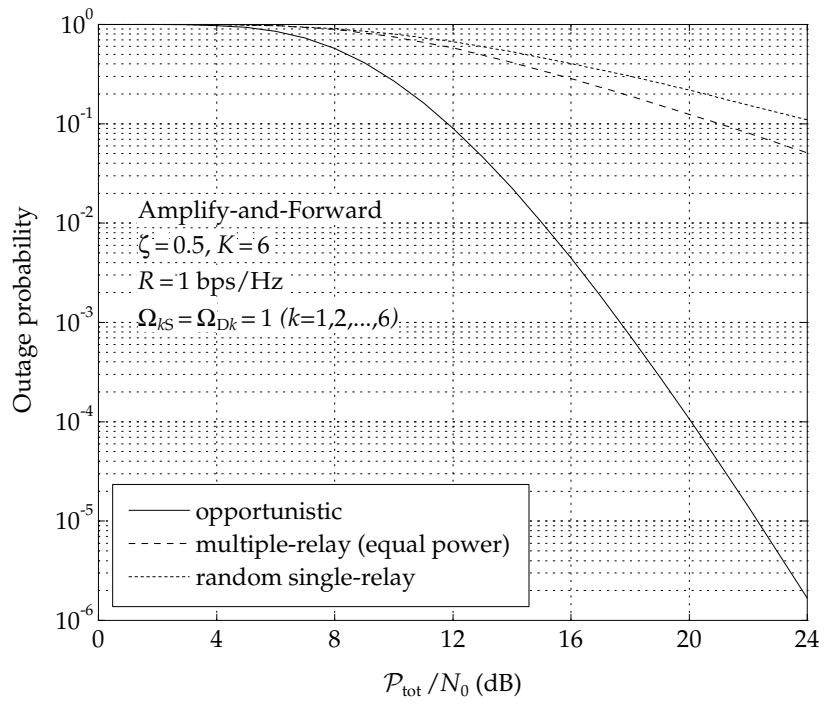

Fig. 7. Outage probability as a function of $\mathcal{P}_{\text {tot }} / N_{0}$ for the AaF strategy at the end-to-end spectral efficiency $R=1 \mathrm{bps} / \mathrm{Hz}$ in symmetric channels. $\zeta=0.5, K=6$, and $\Omega_{\mathrm{S} k}=\Omega_{k \mathrm{D}}=1, k=1,2, \ldots, 6$. Opportunistic relaying is compared with equal-power MR transmissions and random singlerelay selection.

the same average channel gains. For AaF schemes, the mutual information in (24) involves the vectorial addition of multiple relay terms. This is due to the fact that MR transmitted signals do not necessarily add constructively at the receiver, and therefore, their superposition does not increases linearly with the number of relays. Both figures show again that selecting a relay based on average channel gain removes the potential benefits of opportunistic relaying.

Finally, Figs. 9 and 10 show the performance of opportunistic $\mathrm{AaF}$ relaying as a function of $\zeta$ for the symmetric and asymmetric scenarios, respectively. It is shown that $\zeta=0.5$ is not the optimal $\zeta^{*}$, even for the symmetric scenario. This is due to the fact that relays also amplify the noise introduced in the first reception and therefore, it is important at the final destination to overcome noise introduced at the relay as well as at the destination. From that perspective, it is preferable to allocate more power at the opportunistic relay than to the source. The figures also show that choice of $\zeta=0.5$ (in the absence of network CSI) incurs a small performance loss of a few parts-per-million of the outage probability. Therefore, $\zeta=0.5$ is a reasonable choice in practice, since it gives a near-optimal performance.

\section{CONCLUSION}

This paper presented opportunistic relaying protocols and analyzed outage performance under an aggregate power constraint. In particular, we proposed simple opportunistic relaying protocols that can be implemented in distributed manners without requiring global CSI. We showed that both reactive and proactive opportunistic $\mathrm{DaF}$ relaying are outage-optimal. We further showed that opportunistic AaF relaying significantly outperforms AaF strategies based on equal-power MR transmissions, when only local CSI is available. Additionally, opportunistic $\mathrm{AaF}$ relaying is outage-optimal among single relay selection schemes. Finally, we demonstrated that equal

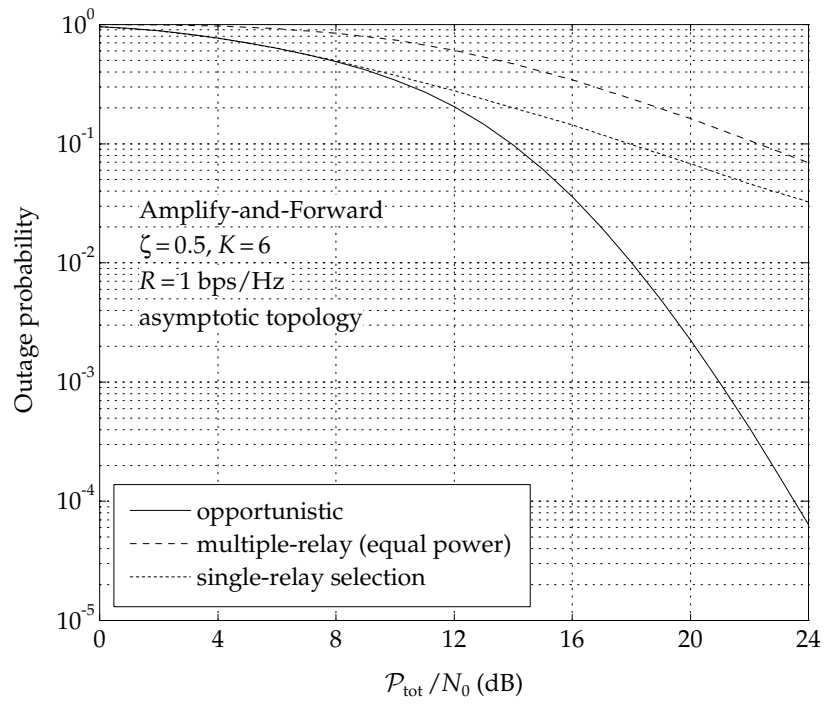

Fig. 8. Outage probability as a function of $\mathcal{P}_{\text {tot }} / N_{0}$ for the AaF strategy at the end-to-end spectral efficiency $R=1 \mathrm{bps} / \mathrm{Hz}$ in asymmetric channels. $\zeta=$ $0.5, K=6$, and $\left\{\Omega_{\mathrm{S} k}\right\}_{k=1}^{K}=\left\{\Omega_{k \mathrm{D}}\right\}_{k=1}^{K}=\{4.5,0.5,0.4,0.3,0.2,0.1\}$. Opportunistic relaying is compared with equal-power MR transmissions and single-relay selection based on average channel gains.

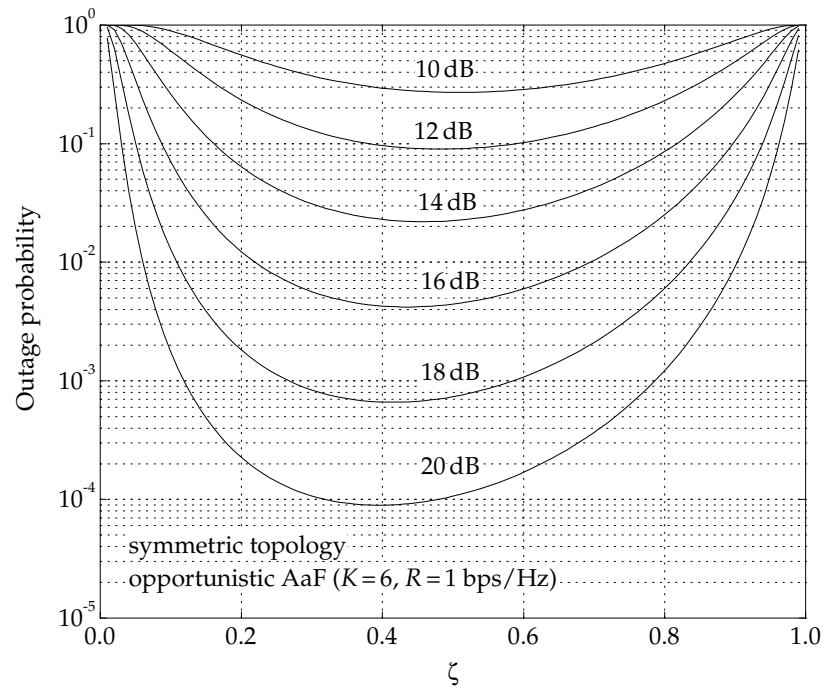

Fig. 9. Outage probability as a function of power allocation $\zeta$ for opportunistic $\mathrm{AaF}$ relaying at the end-to-end spectral efficiency $R=1 \mathrm{bps} / \mathrm{Hz}$ in symmetric channels when $\mathcal{P}_{\text {tot }} / N_{0}=10,12,14,16,18$, and $20 \mathrm{~dB}$. $K=6$ and $\Omega_{\mathrm{S} k}=\Omega_{k \mathrm{D}}=1, k=1,2, \ldots, 6$. The optimal value of $\zeta$ is not equal to $\zeta^{*}=0.5$ for all values of $\mathcal{P}_{\text {tot }} / N_{0}$.

power allocation between the source and opportunistic relay gives a near-optimal performance without requiring global CSI.

Proactive opportunistic relaying allows all relays, except a single opportunistic relay, to enter an idle mode even during the source transmission, which reduces the reception energy cost in the network. Therefore, our proactive strategy can be viewed as energy-efficient routing in the network. In contrast, the reactive schemes require all relays to receive information during the source transmission and consequently, scale the reception energy proportionally with the network size. This overhead may not be negligible, especially in battery-operated terminals. 


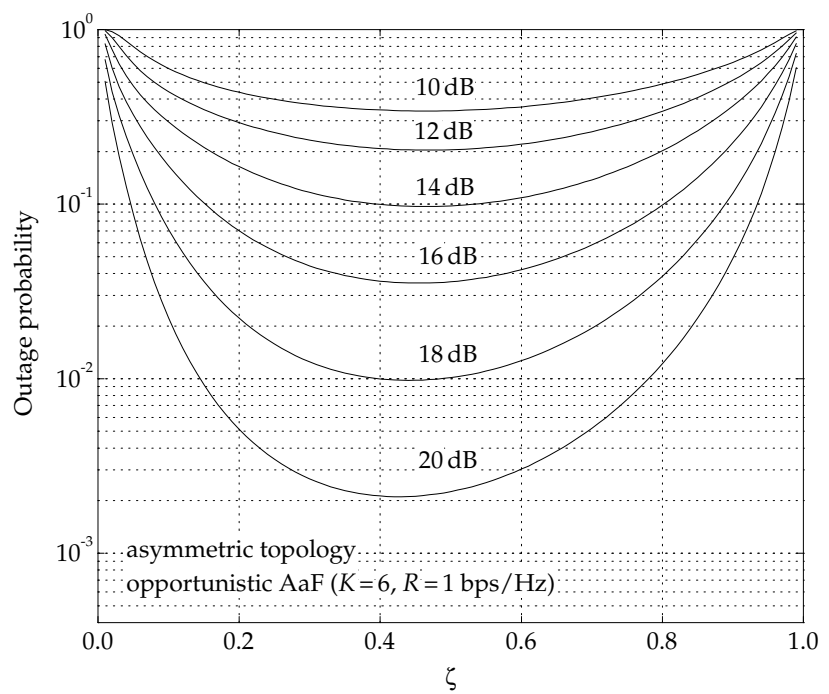

Fig. 10. Outage probability as a function of power allocation $\zeta$ for opportunistic AaF relaying at the end-to-end spectral efficiency $R=1 \mathrm{bps} / \mathrm{Hz}$ in asymmetric channels when $\mathcal{P}_{\text {tot }} / N_{0}=10,12,14,16,18$, and $20 \mathrm{~dB}$. $K=6$ and $\left\{\Omega_{\mathrm{S} k}\right\}_{k=1}^{K}=\left\{\Omega_{k \mathrm{D}}\right\}_{k=1}^{K}=\{4.5,0.5,0.4,0.3,0.2,0.1\}$. The optimal value of $\zeta$ is not equal to $\zeta^{*}=0.5$ for all values of $\mathcal{P}_{\text {tot }} / N_{0}$.

Our results reveal that relays in cooperative communications can be viewed not only as active re-transmitters, but also as distributed sensors of the wireless channel. Cooperative relays can be useful even when they do not transmit, provided that they cooperatively listen. In that way, cooperation benefits can be cultivated with simple radio implementation.

\section{APPENDIX}

\section{SOME DisTributions INVOLVING EXPONENTIAL VARIATES}

\section{A. A Sum Distribution}

Theorem 2 (Sum Distribution): Let $Y_{n} \sim \Upsilon\left(1 / \mu_{n}\right), n=$ $1,2, \ldots, N$, be $N$ statistically independent and not necessarily identically distributed (i.n.i.d.) exponential r.v.'s. Then, the p.d.f. of a sum $X=\sum_{n=1}^{N} Y_{n}$ is given by

$$
p_{X}(x)=\sum_{i=1}^{\varrho(\mathcal{A})} \sum_{j=1}^{\tau_{i}(\boldsymbol{A})} \mathcal{X}_{i, j}(\mathcal{A}) \frac{\mu_{\langle i\rangle}^{-j}}{(j-1) !} x^{j-1} e^{-x / \mu_{\langle i\rangle}}, x \geq 0
$$

where $\mathcal{A}=\operatorname{diag}\left(\mu_{1}, \mu_{2}, \ldots, \mu_{N}\right), \varrho(\mathcal{A})$ is the number of distinct diagonal elements of $\mathcal{A}, \mu_{\langle 1\rangle}>\mu_{\langle 2\rangle}>\ldots>\mu_{\langle\varrho(\mathcal{A})\rangle}$ are the distinct diagonal elements in decreasing order, $\tau_{i}(\mathcal{A})$ is the multiplicity of $\mu_{\langle i\rangle}$, and $\mathcal{X}_{i, j}(\mathcal{A})$ is the $(i, j)$ th characteristic coefficient of $\mathcal{A}$ [23]. ${ }^{13}$ The cumulative distribution function (c.d.f.) of $X$ is given by

$F_{X}(x)=1-\sum_{i=1}^{\varrho(\mathcal{A})} \sum_{j=1}^{\tau_{i}(\boldsymbol{\mathcal { A }})} \sum_{k=0}^{j-1} \frac{\mathcal{X}_{i, j}(\mathcal{A})}{k !}\left(\frac{x}{\mu_{\langle i\rangle}}\right)^{k} e^{-x / \mu_{\langle i\rangle}}, x \geq 0$

Proof: Since $Y_{1}, Y_{2}, \ldots, Y_{N}$ are statistically independent, the characteristic function (c.f.) of $X$ is

$$
\Phi_{X}(\jmath w) \triangleq \mathbb{E}\left\{e^{\jmath w X}\right\}=\prod_{n=1}^{N}\left(1-\jmath w \mu_{n}\right)^{-1}
$$

Using a partial fraction decomposition of (31) with the characteristic coefficients, we obtain the p.d.f. of $X$ as

$$
\begin{aligned}
p_{X}(x) & =\frac{1}{2 \pi} \int_{-\infty}^{\infty} e^{-\jmath w x} \Phi_{X}(\jmath w) d w \\
& =\frac{1}{2 \pi} \int_{-\infty}^{\infty} e^{\jmath w x} \operatorname{det}\left(\mathbf{I}_{N}+\jmath w \mathcal{A}\right)^{-1} d w \\
& =\sum_{i=1}^{\varrho(\mathcal{A})} \sum_{j=1}^{\tau_{i}(\mathcal{A})} \frac{\mathcal{X}_{i, j}(\mathcal{A})}{2 \pi} \int_{-\infty}^{\infty} e^{\jmath w x}\left(1+\jmath w \mu_{\langle i\rangle}\right)^{-j} d w \\
& =\sum_{i=1}^{\varrho(\mathcal{A})} \sum_{j=1}^{\tau_{i}(\mathcal{A})} \mathcal{X}_{i, j}(\mathcal{A}) \frac{\mu_{\langle i\rangle}^{-j}}{(j-1) !} x^{j-1} e^{-x / \mu_{\langle i\rangle}} u(x)
\end{aligned}
$$

where $\mathbf{I}_{N}$ denotes the $N \times N$ identity matrix and $u(x)$ is the Heaviside step function.

From (32), we obtain the c.d.f. of $X$ as

$$
\begin{aligned}
F_{X}(x) & =\sum_{i=1}^{\varrho(\mathcal{A})} \sum_{j=1}^{\tau_{i}(\mathcal{A})} \mathcal{X}_{i, j}(\mathcal{A}) \frac{\mu_{\langle i\rangle}^{-j}}{(j-1) !} \int_{0}^{x} t^{j-1} e^{-t / \mu_{\langle i\rangle}} d t \\
& =\sum_{i=1}^{\varrho(\mathcal{A})} \sum_{j=1}^{\tau_{i}(\mathcal{A})} \mathcal{X}_{i, j}(\mathcal{A})\left\{1-\frac{1}{(j-1) !} \Gamma\left(j, \frac{x}{\mu_{\langle i\rangle}}\right)\right\} \\
& =1-\sum_{i=1}^{\varrho(\mathcal{A})} \sum_{j=1}^{\tau_{i}(\mathcal{A})} \frac{\mathcal{X}_{i, j}(\mathcal{A})}{(j-1) !} \Gamma\left(j, \frac{x}{\mu_{\langle i\rangle}}\right)
\end{aligned}
$$

where the last equality follows from the fact that the sum of all the characteristic coefficients is equal to one [23], and $\Gamma(n, z)$ is the incomplete gamma function defined by

$$
\Gamma(n, z) \triangleq \int_{z}^{\infty} t^{n-1} e^{-t} d t
$$

Finally, using the identity [26, eq. (8.352.2)]

$$
\Gamma(n, z)=(n-1) ! e^{-z} \sum_{k=0}^{n-1} \frac{z^{k}}{k !}, \quad n \text { positive integer }
$$

yields the desired result (30).

${ }^{13}$ Let $\boldsymbol{\Sigma}$ be an $n \times n$ Hermitian matrix with the eigenvalues $\sigma_{1}, \sigma_{2}, \ldots, \sigma_{n}$ in any order, $\varrho(\boldsymbol{\Sigma})$ be the number of distinct eigenvalues, $\left.\sigma_{\langle 1\rangle}>\sigma_{\langle 2\rangle}\right\rangle$ $\ldots>\sigma_{\langle\varrho(\boldsymbol{\Sigma})\rangle}$ be the distinct eigenvalues in decreasing order, and $\tau_{i}(\boldsymbol{\Sigma})$ be the multiplicity of $\sigma_{\langle i\rangle}$. Then, the $(i, j)$ th characteristic coefficient $\mathcal{X}_{i, j}(\boldsymbol{\Sigma})$, $i=1,2, \ldots, \varrho(\boldsymbol{\Sigma}), j=1,2, \ldots, \tau_{i}(\boldsymbol{\Sigma})$, is defined as a partial fraction expansion coefficient of $\operatorname{det}\left(\mathbf{I}_{n}+\xi \boldsymbol{\Sigma}\right)^{-1}$ such that

$$
\begin{aligned}
\operatorname{det}\left(\mathbf{I}_{n}+\xi \boldsymbol{\Sigma}\right)^{-1} & =\prod_{i=1}^{\varrho(\boldsymbol{\Sigma})}\left(1+\xi \sigma_{\langle i\rangle}\right)^{-\tau_{i}(\boldsymbol{\Sigma})} \\
& =\sum_{i=1}^{\varrho(\boldsymbol{\Sigma})} \sum_{j=1}^{\tau_{i}(\boldsymbol{\Sigma})} \mathcal{X}_{i, j}(\boldsymbol{\Sigma})\left(1+\xi \sigma_{\langle i\rangle}\right)^{-j}
\end{aligned}
$$

. where $\xi$ is a scalar constant such that $\mathbf{I}_{n}+\xi \boldsymbol{\Sigma}$ is nonsingular and $\mathcal{X}_{i, j}(\boldsymbol{\Sigma})$ can be determined by

$$
\begin{aligned}
\mathcal{X}_{i, j}(\boldsymbol{\Sigma})= & \frac{1}{\varpi_{i, j} ! \sigma_{\langle i\rangle}^{\varpi_{i, j}}} \\
& \times\left.\left[\frac{d^{\varpi_{i, j}}}{d v^{\varpi_{i, j}}}\left(1+v \sigma_{\langle i\rangle}\right)^{\tau_{i}(\boldsymbol{\Sigma})} \operatorname{det}\left(\mathbf{I}_{n}+v \boldsymbol{\Sigma}\right)^{-1}\right]\right|_{v=-\frac{1}{\sigma_{\langle i\rangle}}}
\end{aligned}
$$

with $\varpi_{i, j}=\tau_{i}(\boldsymbol{\Sigma})-j$. 
The following corollaries are two extreme cases of Theorem 2 , i.e., the cases of all distinct $\mu_{n}$ 's and all equal $\mu_{n}$ 's.

Corollary 1: If all of $\mu_{n}$ 's are distinct $(\varrho(\mathcal{A})=N$ and $\tau_{i}(\mathcal{A})=1$ in Theorem 2), then we have

$$
p_{X}(x)=\sum_{i=1}^{N}\left\{\prod_{\substack{j=1 \\ j \neq i}}^{N}\left(1-\frac{\mu_{j}}{\mu_{i}}\right)^{-1}\right\} \frac{e^{-x / \mu_{i}}}{\mu_{i}}, \quad x \geq 0
$$

and

$$
F_{X}(x)=1-\sum_{i=1}^{N}\left\{\prod_{\substack{j=1 \\ j \neq i}}^{N}\left(1-\frac{\mu_{j}}{\mu_{i}}\right)^{-1}\right\} e^{-x / \mu_{i}}, \quad x \geq 0 .
$$

Proof: When all of $\mu_{n}$ 's are distinct, the characteristic coefficients of $\mathcal{A}$ become [23]

$$
\mathcal{X}_{i, 1}(\mathcal{A})=\prod_{\substack{j=1 \\ j \neq i}}^{N}\left(1-\frac{\mu_{j}}{\mu_{i}}\right)^{-1}, \quad i=1,2, \ldots, N
$$

The proof follows immediately from Theorem 2 and (38).

Corollary 2: If $\mu_{n}=\mu, n=1,2, \ldots, N(\varrho(\mathcal{A})=1$ and $\tau_{1}(\mathcal{A})=N$ in Theorem 2), then we have

$$
p_{X}(x)=\frac{\mu^{-N}}{(N-1) !} x^{N-1} e^{-x / \mu}, \quad x \geq 0
$$

and

$$
F_{X}(x)=1-\sum_{k=0}^{N-1} \frac{1}{k !}\left(\frac{x}{\mu}\right)^{k} e^{-x / \mu}, \quad x \geq 0 .
$$

Proof: When all of $\mu_{n}$ 's are equal, the characteristic coefficients of $\mathcal{A}$ become [23]

$$
\mathcal{X}_{1, j}(\mathcal{A})= \begin{cases}0, & j=1,2, \ldots, N-1 \\ 1, & j=N\end{cases}
$$

The proof follows immediately from Theorem 2 and (41).

We remark that Corollary 2 agrees with the well-known fact that a sum of $N$ i.i.d. exponential r.v.'s has a central chisquared distribution with $2 N$ degrees of freedom.

\section{B. A Product-Ratio Distribution}

Theorem 3 (Product-Ratio Distribution): Let

$$
\begin{aligned}
& Y_{1} \sim \Upsilon\left(1 / \mu_{1}\right) \\
& Y_{2} \sim \Upsilon\left(1 / \mu_{2}\right)
\end{aligned}
$$

be statistically i.n.i.d. exponential r.v.'s. Suppose a productratio $X$ of the form

$$
X=\frac{Y_{1} Y_{2}}{a \mu_{1}+Y_{2}}, \quad a>0 .
$$

Then, we have

$$
p_{X}(x)=\frac{1}{\mu_{1} \mu_{2}} \int_{0}^{\infty} \frac{a \mu_{1}+z}{z} \exp \left\{-\frac{x\left(a \mu_{1}+z\right)}{\mu_{1} z}-\frac{z}{\mu_{2}}\right\} d z
$$

and

$$
F_{X}(x)=1-\frac{1}{\mu_{2}} \int_{0}^{\infty} \exp \left\{-\frac{x\left(a \mu_{1}+z\right)}{\mu_{1} z}-\frac{z}{\mu_{2}}\right\} d z
$$

where $x \geq 0$.

Proof: Note that

$$
\begin{aligned}
F_{X}(x) & =\operatorname{Pr}\left\{\frac{Y_{1} Y_{2}}{a \mu_{1}+Y_{2}} \leq x\right\} \\
& =\mathbb{E}_{Y_{2}}\left\{F_{X \mid Y_{2}}(x)\right\} \\
& =\mathbb{E}_{Y_{2}}\left\{1-\exp \left[-\frac{x\left(a \mu_{1}+Y_{2}\right)}{\mu_{1} Y_{2}}\right]\right\} \\
& =1-\frac{1}{\mu_{2}} \int_{0}^{\infty} \exp \left\{-\frac{x\left(a \mu_{1}+z\right)}{\mu_{1} z}-\frac{z}{\mu_{2}}\right\} d z
\end{aligned}
$$

as desired. The p.d.f. of $X$ in (43) follows immediately from differentiating (44) with respect to $x$.

\section{REFERENCES}

[1] J. H. Winters, "On the capacity of radio communication systems with diversity in Rayleigh fading environment," IEEE J. Select. Areas Commun., vol. 5, no. 5, pp. 871-878, June 1987.

[2] A. Sendonaris, E. Erkip, and B. Aazhang, "User cooperation diversityPart I: System description," IEEE Trans. Commun., vol. 51, no. 11, pp. 1927-1938, Nov. 2003.

[3] J. N. Laneman, D. N. C. Tse, and G. W. Wornell, "Cooperative diversity in wireless networks: Efficient protocols and outage behavior,' IEEE Trans. Inform. Theory, vol. 50, no. 12, pp. 3062-3080, Dec. 2004.

[4] R. U. Nabar, H. Bölcskei, and F. W. Kneubühler, "Fading relay channels: Performance limits and space-time signal design," IEEE J. Select. Areas Commun., vol. 22, no. 6, pp. 1099-1109, Aug. 2004.

[5] G. Kramer, M. Gastpar, and P. Gupta, "Cooperative strategies and capacity theorems for relay networks," IEEE Trans. Inform. Theory, vol. 51, no. 9, pp. 3037-3063, Sep. 2005.

[6] M. Gastpar and M. Vetterli, "On the capacity of large Gaussian relay networks," IEEE Trans. Inform. Theory, vol. 51, no. 3, pp. 765-779, Mar. 2005.

[7] J. N. Laneman and G. W. Wornell, "Distributed space-time coded protocols for exploiting cooperative diversity in wireless networks," IEEE Trans. Inform. Theory, vol. 49, no. 10, pp. 2415-2525, Oct. 2003.

[8] X.-B. Liang and X.-G. Xia, "Unitary signal constellations for differential space-time modulation with two transmit antennas: Parametric codes, optimal designs, and bounds," IEEE Trans. Inform. Theory, vol. 48, no. 8, pp. 2291-2322, Aug. 2002.

[9] H. Wang and X.-G. Xia, "Upper bounds of rates of complex orthogonal space-time block codes," IEEE Trans. Inform. Theory, vol. 49, no. 10, pp. 2788-2796, Oct. 2003.

[10] K. Lu, S. Fu, and X.-G. Xia, "Closed form designs of complex orthogonal space-time block codes of rates $(k+1) /(2 k)$ for $2 k-1$ or $2 k$ transmit antennas," IEEE Trans. Inform. Theory, vol. 51, no. 12, pp. 4340-4347, Dec. 2005.

[11] P. Larsson and H. Rong, "Large-scale cooperative relay network with optimal coherent combining under aggregate relay power constraints," in Proc. Working Group 4, World Wireless Research Forum WWRF8 Meeting, Feb. 2004.

[12] Y. Jing and B. Hassibi, "Distributed space-time coding in wireless relay networks," IEEE Trans. Wireless Commun., vol. 5, no. 12, pp. 35243536, Dec. 2006.

[13] I. Maric and R. D. Yates, "Bandwidth and power allocation for cooperative strategies in Gaussian relay networks," in Proc. Asilomar Conf. Signals, Syst., Computers, Nov. 2004, pp. 1907-1911.

[14] M. Z. Win and J. H. Winters, "Methods and systems for spatial processing," U.S. Patent 6,804,312, Oct. 12, 2004.

[15] —, "Analysis of hybrid selection/maximal-ratio combining in Rayleigh fading," IEEE Trans. Commun., vol. 47, no. 12, pp. 17731776, Dec. 1999.

[16] _ - "Virtual branch analysis of symbol error probability for hybrid selection/maximal-ratio combining in Rayleigh fading," IEEE Trans. Commun., vol. 49, no. 11, pp. 1926-1934, Nov. 2001.

[17] M. Z. Win, N. C. Beaulieu, L. A. Shepp, B. F. Logan, and J. H. Winters, "On the SNR penalty of MPSK with hybrid selection/maximal ratio combining over IID Rayleigh fading channels," IEEE Trans. Commun., vol. 51, no. 6, pp. 1012-1023, June 2003. 
[18] A. F. Molisch and M. Z. Win, "MIMO systems with antenna selectionAn overview," IEEE Microwave Mag., vol. 5, no. 1, pp. 46-56, Mar. 2004.

[19] A. Bletsas, "Intelligent antenna sharing in cooperative diversity wireless networks," Ph.D. dissertation, Massachusetts Institute of Technology, Cambridge, MA, Sep. 2005.

[20] K. Azarian, H. E. Gamal, and P. Schniter, "On the achievable diversityvs-multiplexing tradeoff in cooperative channels," IEEE Trans. Inform. Theory, vol. 51, no. 12, pp. 4152-4172, Dec. 2005.

[21] J. Adeane, M. R. D. Rodrigues, and I. J. Wassell, "Optimum power allocation in cooperative networks," in Proc. Postgraduate Research Conf. Electronics, Photonics, Commun. Networks, Computing Sci., Mar.Apr. 2005, pp. 23-24.

[22] A. Bletsas, A. Khisti, D. P. Reed, and A. Lippman, "A simple cooperative diversity method based on network path selection," IEEE J. Select. Areas Commun., vol. 24, no. 3, pp. 659-672, Mar. 2006.

[23] H. Shin and M. Z. Win, "MIMO diversity in the presence of double scattering," IEEE Trans. Inform. Theory, revised for publication. [Online]. Available: http://arxiv.org/abs/cs.IT/0511028

[24] J. Luo, R. S. Blum, L. J. Cimini, L. J. Greenstein, and A. M. Haimovich, "Link-failure probabilities for practical cooperative relay networks," in Proc. IEEE VTC-Spring, June 2005, pp. 1489-1493.

[25] B. Zhao and M. C. Valenti, "Practical relay networks: A generalization of hybrid-ARQ," IEEE J. Select. Areas Commun., vol. 23, no. 1, pp. 7-18, Jan. 2005

[26] I. S. Gradshteyn and I. M. Ryzhik, Tables of Integrals, Series, and Products, 6th ed. San Diego, CA: Academic Press, Inc., 2000.

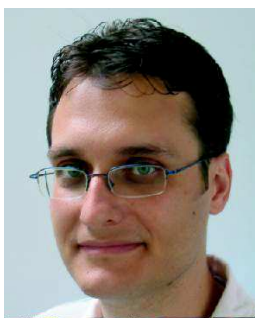

Aggelos Bletsas (S'03-M'05) received with excellence his diploma degree in Electrical and Computer Engineering from Aristotle University of Thessaloniki, Greece in 1998. He worked as a Research Assistant from 1999 to 2005 at the Massachusetts Institute of Technology (MIT) Media Laboratory, where he earned the S.M. and Ph.D. degrees in 2001 and 2005, respectively. He worked at Mitsubishi Electric Research Laboratories (MERL) as an intern during the summer of 2004 and as a Postdoctoral Fellow from fall of 2005 to fall of 2006 , when he returned to Greece to serve in the Greek Army. Upon completion of his military service in May 2007, he joined the Radio Communications Laboratory (RCL), Department of Physics, Aristotle University of Thessaloniki, as a visiting scientist.

His research interests span the broad area of scalable wireless communication and networking, with emphasis on relay techniques, signal processing for communication, radio hardware/software implementations for wireless transceivers and low cost sensor networks, time/frequency metrology and nanotechnology. He is currently researching and developing detection and signal processing techniques for the long-range backscatter wireless channel.

He received best thesis award in 1999 from Ericsson, for the development of the first, complete and publicly available Hellenic text-to-speech system (called Esopos). He earned several awards for academic excellence from the National Scholarship Foundation, Greece and the Technical Chamber of Greece. He was a recipient of a BT Fellowship Award and a Nortel Networks Fellowship Award from 2000 to 2005, during his graduate studies at MIT.

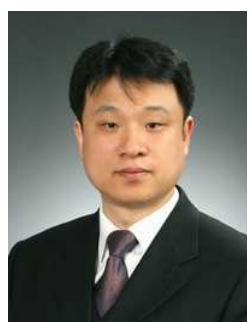

Hyundong Shin (S'01-M'04) received the B.S. degree in Electronics Engineering from Kyung Hee University, Korea, in 1999, and the M.S. and Ph.D. degrees in Electrical Engineering from Seoul National University, Seoul, Korea, in 2001 and 2004, respectively.

From September 2004 to February 2006, Dr. Shin was a Postdoctoral Associate at the Laboratory for Information and Decision Systems (LIDS), Massachusetts Institute of Technology (MIT), Cambridge, MA, USA. In March 2006, he joined the faculty of the School of Electronics and Information, Kyung Hee University, Korea, where he is now an Assistant Professor at the Department of Radio Communication Engineering. His research interests include wireless communications, information and coding theory, cooperative/collaborative communications, and multiple-antenna wireless communication systems and networks.

Prof. Shin served on the Technical Program Committees for the 2006 IEEE International Conference on Communications (ICC'06) and the 2006 IEEE International Conference on Ultra Wideband (ICUWB'06). He currently serves as an Editor for the IEEE TRANSACTIONS ON WIRELESS COMMUNICATIONS. He is a Guest Editor for the 2007 EURASIP Journal on Advances in Signal Processing (Special Issue on Wireless Cooperative Networks).

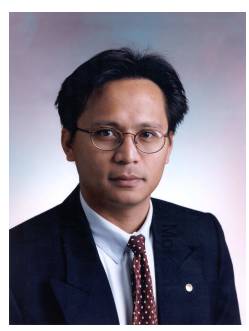

Moe Z. Win (S'85-M'87-SM'97-F'04) received the B.S. degree (magna cum laude) from Texas A\&M University, College Station, in 1987 and the M.S. degree from the University of Southern California (USC), Los Angeles, in 1989, both in Electrical Engineering. As a Presidential Fellow at USC, he received both an M.S. degree in Applied Mathematics and the Ph.D. degree in Electrical Engineering in 1998.

Dr. Win is an Associate Professor at the Laboratory for Information \& Decision Systems (LIDS), Massachusetts Institute of Technology (MIT). Prior to joining MIT, he spent five years at AT\&T Research Laboratories and seven years at the Jet Propulsion Laboratory. His main research interests are the applications of mathematical and statistical theories to communication, detection, and estimation problems. Specific current research topics include measurement and modeling of time-varying channels, design and analysis of multiple antenna systems, ultra-wide bandwidth (UWB) systems, optical transmission systems, and space communications systems.

Professor Win has been actively involved in organizing and chairing a number of international conferences. He served as the Technical Program Chair for the IEEE Conference on Ultra Wideband in 2006, the IEEE Communication Theory Symposia of ICC-2004 and Globecom-2000, and the IEEE Conference on Ultra Wideband Systems and Technologies in 2002; Technical Program Vice-Chair for the IEEE International Conference on Communications in 2002; and the Tutorial Chair for the IEEE Semiannual International Vehicular Technology Conference in Fall 2001. He served as a chair (2004-2006) and secretary (2002-2004) for the Radio Communications Committee of the IEEE Communications Society. Dr. Win is currently an Editor for IEEE TRANSACTIONS ON Wireless Communications. He served as Area Editor for Modulation and Signal Design (2003-2006), Editor for Wideband Wireless and Diversity (2003-2006), and Editor for Equalization and Diversity (1998-2003), all for the IEEE TRANSACTIONS ON COMMUNiCATIONS. He was Guest-Editor for the 2002 IEEE JOURNAL ON SELECTED AREAS In COMMUNICATIONS (Special Issue on Ultra -Wideband Radio in Multiaccess Wireless Communications).

Professor Win received the International Telecommunications Innovation Award from Korea Electronics Technology Institute in 2002, a Young Investigator Award from the Office of Naval Research in 2003, and the IEEE Antennas and Propagation Society Sergei A. Schelkunoff Transactions Prize Paper Award in 2003. In 2004, Dr. Win was named Young Aerospace Engineer of the Year by AIAA, and garnered the Fulbright Foundation Senior Scholar Lecturing and Research Fellowship, the Institute of Advanced Study Natural Sciences and Technology Fellowship, the Outstanding International Collaboration Award from the Industrial Technology Research Institute of Taiwan, and the Presidential Early Career Award for Scientists and Engineers from the United States White House. He was honored with the 2006 IEEE Eric E. Sumner Award "for pioneering contributions to ultra-wide band communications science and technology." Professor Win is an IEEE Distinguished Lecturer and elected Fellow of the IEEE, cited "for contributions to wideband wireless transmission." 